Abstracted/indexed in Academic Search Complete, Asia Journals Online, Bangladesh Journals Online, Biological Abstracts, BIOSIS Previews, CAB Abstracts, Current Abstracts, Directory of Open Access Journals, EMBASE/Excerpta Medica, Google Scholar, HINARI (WHO), International Pharmaceutical Abstracts, Open J-gate, Science Citation Index Expanded, SCOPUS and Social Sciences Citation Index;

ISSN: $1991-0088$

\title{
Cytotoxicity study of dimethylisatin and its heterocyclic derivatives
}

\author{
Mohammad Mamun Hossain, Nazrul Islam, Rahat Khan and Md. Rabiul Islam
}

Department of Chemistry, Jahangirnagar University, Savar, Dhaka 1342, Bangladesh.

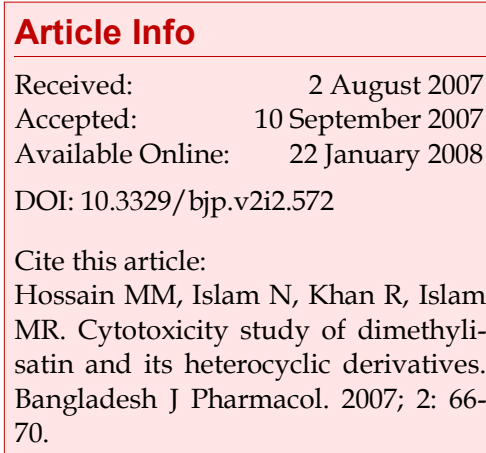

\begin{abstract}
Isatin derivatives are bioactive molecules. To study the cytotoxicity and eventually the anticancer activities against cancer cell lines, a series of dimethyl-substitituted isatin derivatives (4-8) starting from isatin thiosemicarbazones (3) had been synthesized in high yields. Investigation of the cytotoxicity of these compounds was carried out against brine shrimp by lethality bioassay. The present study shows that compounds 4, 5, 6 and 8' with heterocyclic moiety had pronounced cytotoxicity whereas 7, 7' and 8 were moderately active. It is remarkable that the substituent, $\mathrm{X}=-\mathrm{OCH}_{3}$ has greater activity than the bromine atom in this series.
\end{abstract}

\section{Introduction}

Isatin chemically known as $1 \mathrm{H}$-indole-2,3-dione has become a popular topic due to its manifold uses. The chemistry of isatin and its derivatives is particularly interesting because of their potential application in medicinal chemistry. Isatins are very important compounds due to their antifungal properties (Islam et al., 1998). Moreover, isatins are the synthetic precursors of some biologically important heterocyclic compounds such as quinoline 1,3,4-thiadiazolines etc (Ram et al., 1980; Raj et al., 2003). The biological activity of $\Delta^{2}-1,3,4-$ thiadiazoline heterocycles can be obtained from the corresponding schiff bases of isatins (Islam et al., 2000, 2001, 2003). Besides, the triazins [5, 6-b] indole-3-thione derivatives have attracted considerable attention in the field of medicine due to their antifungal, antimalarial and antiparasitic properties (Pal et al., 1991). Over the last five decades the considerable research effort has been devoted to the field of HIV and AIDS, a fatal disease, to develop or immunosuppressive agents across the world. Surprisingly isatin derivatives show anti-HIV activity too (Silvam et al., 2001). Therefore, the research interest on isatins has expended day to day. Here some important heterocyclic derivatives of isatins and consequent study of their cytotoxicity are reported.

\section{Materials and Methods}

The melting points of the synthesized compounds were recorded by thin disc method on a Fischer Jonhns electro -thermal melting point apparatus. Infrared spectra were recorded on DR-8001, Shimadzu FT-IR spectrophotometer, ${ }^{1} \mathrm{H}-\mathrm{NMR}$ spectra on a WP-400 NMR spectrophotometer using tetramethylsilane as internal standard. Mass spectra were obtained on a Kratas MS-25 using DH-88 data system.

The tested compound (Scheme 1) 2, 3, 4, 5, 6, 7, 7', 8 and 8 ' were synthesized by the standard procedure. The characterization of synthesized compounds was carried out by IR, ${ }^{1} \mathrm{H}-\mathrm{NMR}$ and mass spectroscopic methods (Table I). According to standard sandmeyer procedure (Kondrashova and Shvekhgeimer, 2002) oximinoacetanilide 1 was synthesized from 2,6-dimethyl aniline by using chloral hydrate. The resulting pale yellow solid was cyclized with concentrated $\mathrm{H}_{2} \mathrm{SO}_{4}$ under warm condition to give the corresponding dimethylisatin 2 as a red solid in high yield. Compound 2 was treated with thiosemicarbazide in glacial acetic acid under refluxing 
<smiles>Cc1cccc(NC(=O)C=NO)c1C</smiles>

1<smiles>CC(=O)NC1=NN(C(C)=O)C2(S1)C(=O)Nc1c2ccc(C)c1C</smiles>

4<smiles>Cc1ccc2c(c1C)NC(=O)C2=O</smiles>

2<smiles>Cc1ccc2c([nH]c3nc(=S)[nH]nc32)c1C</smiles>

5<smiles>Cc1ccc2c(c1C)NC(=O)/C2=N/NC(N)=S</smiles>

3

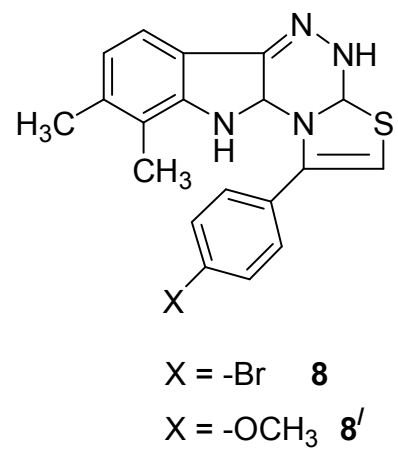

Scheme 1

condition for 3 hours. Cooling followed by neutraliztion of the reaction mixture gave a crude solid, which was recrystallized from methanol to give 3 as yellow crystals in high yield (Table II). Refluxing of compound 3 with acetic anhydride gave a crude solid mass, which was recrystallized from methanol to give 4 as a yellow solid in high yield. On the other hand, alkali $(4 \% \mathrm{KOH})$ mediated refluxing of compound 3 for 3 hours gave compound $\mathbf{5}$ as orange crystals in moderate yield. The ring extended compound 6 was obtained in high yield from 5 with the treatment of 1,2-dibromoethane in DMF. Besides, the treatment of compound 3 with the derivatives of phenacylbromide in a mixture of ethanol and DMF gave the corresponding phenylthiazoles 7 and $7^{\prime}$ in very good yields. Finally $\mathrm{POCl}_{3}$ mediated dehydration of 7 and 7 ' gave thiazol-triazinoindoles 8 and 8 respectively in excellent yield.

\section{Cytotoxicity studies}

The cytotoxicity study of the synthesized compounds was investigated on brine shrimp as a test organism for convenience (Solis et al., 1993). $1.6 \mathrm{mg}$ of each of the compounds was taken in the corresponding sample vials with $1.6 \mathrm{~mL}$ of dimethyl sulfoxide to prepare stock solution. From this stock solution 33, 99, 132 and 165 ppm of each compounds were placed in separate test tubes by microsyringe, $1 \mathrm{~mL}$ of extra dimethyl sulfoxide was given in each test tube with 10-12 brine shrimp. After 1, 2, 3 and 4 hours the test tubes were observed and the number of survived naupli in each test tube was counted and results were noted. From this the percentage of lethality of brine shrimp naupli was calculated at each concentration for each sample. Then the $\mathrm{LD}_{50}$ values were calculated by plotting percentage of mortality against time (Figure 1). $\mathrm{LD}_{50}$ of an agent is the dose, which will kill, or inactive $50 \%$ of the test animal. By this method the $\mathrm{LD}_{50}$ values for all the synthesized compounds were obtained (Table III). 


\section{Table I}

\section{Spectroscopic data of the synthesized compounds}

\begin{tabular}{|c|c|}
\hline Compounds & $\mathrm{IR}\left(\mathrm{cm}^{-1}\right)$ \\
\hline 1 & $\begin{array}{l}3320(\mathrm{OH}), 3352(\mathrm{NH}), 3060(\mathrm{Ph}- \\
\mathrm{H}), 3020 \\
(=\mathrm{CH}), 2920,2864\left(\mathrm{CH}_{3}\right), 1655 \\
(\mathrm{C}=\mathrm{O}, \text { amide }), 1612(\mathrm{C}=\mathrm{N}) \\
1551,1473(\mathrm{C}=\mathrm{C})\end{array}$ \\
\hline 2 & $\begin{array}{l}3195(\mathrm{NH}), 3090(\mathrm{Ph}-\mathrm{H}), 2942, \\
2880\left(\mathrm{CH}_{3}\right), 1736(\mathrm{C}=\mathrm{O}, \text { lactum }), \\
1618(\mathrm{C}=\mathrm{O}, \text { Keto }), 1597,1505 \\
(\mathrm{C}=\mathrm{C} \text {, aromatic })\end{array}$ \\
\hline 3 & $\begin{array}{l}3155,3020\left(\mathrm{NH}_{2}\right), 3067(\mathrm{Ph}-\mathrm{H}), \\
2937,2885(\mathrm{CH}, \text { Saturated }), 1695 \\
(\mathrm{C}=\mathrm{O}, \text { lactum }), 1610,1489(\mathrm{C}=\mathrm{C}, \\
\text { aromatic) }\end{array}$ \\
\hline 4 & $\begin{array}{l}3175(\mathrm{NH}), 3090(\mathrm{Ph}-\mathrm{H}), 2920, \\
2875(\mathrm{CH}, \text { Saturated }), 1742(\mathrm{C}=\mathrm{O}, \\
\text { acetyl), } 1693(\mathrm{C}=\mathrm{O}, \text { lactum }), 1616 \text {, } \\
1499(\mathrm{C}=\mathrm{C}, \text { aromatic })\end{array}$ \\
\hline
\end{tabular}

$53205(\mathrm{NH}), 3060(\mathrm{CH}$, aromatic $)$, 2925, 2865 (CH, saturated), 1595, 1489 (C=C, aromatic), $1142(\mathrm{C}=\mathrm{S})$

$63090(\mathrm{CH}$, aromatic $), 2925,2855$ $(\mathrm{CH}$, saturated $), 1616(\mathrm{C}=\mathrm{N})$, 1689,1487 (C=C, aromatic)

11.10 (s, 1H, NHCO), 7.3 (d, 1H, Jo J =7.55 $\mathrm{Hz}, \mathrm{Ar}-\mathrm{H}), 6.8\left(\mathrm{~d}, 1 \mathrm{H}, J_{o}=7.55 \mathrm{~Hz}, \mathrm{Ar}-\mathrm{H}\right)$, $2.20\left(\mathrm{~s}, 3 \mathrm{H}, \mathrm{CH}_{3}\right), 1.95\left(\mathrm{~s}, 3 \mathrm{H}, \mathrm{CH}_{3}\right)$

$12.84(\mathrm{~s}, 1 \mathrm{H}, \mathrm{NHCO}), 11.50$ (s, 1H, NHCS), $10.40\left(\mathrm{~s}, 1 \mathrm{H}, \mathrm{CS}-\mathrm{NH}_{2}\right), 7.25(\mathrm{~d}, 1 \mathrm{H}$, $J=7.64 \mathrm{~Hz}, \mathrm{Ar}-\mathrm{H}), 6.89$ (d, 1H, J=7.64 Hz, $\mathrm{Ar}-\mathrm{H}), 2.20\left(\mathrm{~s}, 3 \mathrm{H}, \mathrm{CH}_{3}\right), 2.02(\mathrm{~s}, 3 \mathrm{H}$, $\left.\mathrm{CH}_{3}\right)$

$12.02(\mathrm{~s}, 1 \mathrm{H}, \mathrm{NHCO}$, lactum), $8.50(\mathrm{~s}, 1 \mathrm{H}$, $\mathrm{NH}-\mathrm{CO}), 7.20(\mathrm{~d}, 1 \mathrm{H}, J=7.64 \mathrm{~Hz}, \mathrm{Ar}-\mathrm{H})$, $6.85(\mathrm{~d}, 1 \mathrm{H}, \mathrm{J}=7.64 \mathrm{~Hz}, \mathrm{Ar}-\mathrm{H}), 2.40(\mathrm{~s}, 3 \mathrm{H}$, $\left.\mathrm{N}-\mathrm{NCOCH}_{3}\right), 2.30(\mathrm{~s}, 3 \mathrm{H}, \mathrm{C}-$

$\left.\mathrm{NHCOCH}_{3}\right), 2.06\left(\mathrm{~s}, 3 \mathrm{H}, \mathrm{CH}_{3}\right), 2.02$ (s, $\left.3 \mathrm{H}, \mathrm{CH}_{3}\right)$

13.20 (s, 1H, NHCS), 10.50 (s, 1H, NHN), 7.20 (d, 1H, J=7.60 Hz, Ar-H), 6.90 (d, $1 \mathrm{H}, \mathrm{J}=7.60 \mathrm{~Hz}, \mathrm{Ar}-\mathrm{H}), 2.20\left(\mathrm{~s}, 3 \mathrm{H}, \mathrm{CH}_{3}\right)$, $2.10\left(\mathrm{~s}, 3 \mathrm{H}, \mathrm{CH}_{3}\right)$

7.25 (d, 1H, J=7.55 Hz, Ar-H), 6.85 (d, $1 \mathrm{H}, J=7.55 \mathrm{~Hz}, \mathrm{Ar}-\mathrm{H}), 4.95\left(\mathrm{t}, 2 \mathrm{H}, \mathrm{CH}_{2-}\right.$ $\mathrm{N}), 3.85\left(\mathrm{t}, 2 \mathrm{H}, \mathrm{CH}_{2}-\mathrm{S}\right), 2.20\left(\mathrm{~s}, 3 \mathrm{H}, \mathrm{CH}_{3}\right)$, $2.15\left(\mathrm{~s}, 3 \mathrm{H}, \mathrm{CH}_{3}\right)$

$73175(\mathrm{NH}), 3060(\mathrm{CH}$, aromatic), 2910, 2840 (CH, saturated), 1693 (C=O, lactum), 1616, 1479 (C=C, aromatic)

$3175(\mathrm{NH}), 3080(\mathrm{CH}$, aromatic), 2920, $2865(\mathrm{CH}$, saturated), 1686 $(\mathrm{C}=\mathrm{O}$, lactum $), 1599,1508(\mathrm{C}=\mathrm{C}$, aromatic)

3095 (CH, aromatic), 2920, 2860 ( $\mathrm{CH}$, saturated), 1616, $1479(\mathrm{C}=\mathrm{C}$, aromatic)

3060 (CH, aromatic), 2920, 2855 (CH, saturated), 1589, $1487(\mathrm{C}=\mathrm{C}$, aromatic)
13.40 (s, 1H, NHCO), 11.45 (s, 1H, NHN), $7.70(\mathrm{~d}, 2 \mathrm{H}, J=8.01 \mathrm{~Hz}, \mathrm{Ar}-\mathrm{H}), 7.40(\mathrm{~d}$, $2 \mathrm{H}, J=8.01 \mathrm{~Hz}, \mathrm{Ar}-\mathrm{H}), 7.20$ (d, 1H, J=7.45 $\mathrm{Hz}, \mathrm{Ar}-\mathrm{H}), 7.10$ (d, 1H, J=7.45 Hz, Ar-H), $7.50(\mathrm{~s}, 1 \mathrm{H},=\mathrm{C}-\mathrm{H}), 2.18\left(\mathrm{~s}, 3 \mathrm{H}, \mathrm{CH}_{3}\right), 2.10$ (s, $\left.3 \mathrm{H}, \mathrm{CH}_{3}\right)$

14.40 (s, 1H, NHCO), 11.30 (s, 1H, NH$\mathrm{N}), 7.90(\mathrm{~d}, 2 \mathrm{H}, \mathrm{J}=8.51 \mathrm{~Hz}, \mathrm{Ar}-\mathrm{H}), 7.25(\mathrm{~d}$, $1 \mathrm{H}, J=7.46 \mathrm{~Hz}, \mathrm{Ar}-\mathrm{H}), 7.15(\mathrm{~d}, 1 \mathrm{H}, J=7.46$ $\mathrm{Hz}, \mathrm{Ar}-\mathrm{H}), 7.20(\mathrm{~s}, 1 \mathrm{H},=\mathrm{C}-\mathrm{H}), 3.60(\mathrm{~s}$, $\left.3 \mathrm{H}, \mathrm{OCH}_{3}\right), 2.20\left(\mathrm{~s}, 3 \mathrm{H}, \mathrm{CH}_{3}\right), 2.10(\mathrm{~s}, 3 \mathrm{H}$, $\mathrm{CH}_{3}$ )

$7.70(\mathrm{~d}, 2 \mathrm{H}, \mathrm{J}=8.56 \mathrm{~Hz}, \mathrm{Ar}-\mathrm{H}), 7.40$ (d, $2 \mathrm{H}, J=8.56 \mathrm{~Hz}, \mathrm{Ar}-\mathrm{H}), 7.20(\mathrm{~d}, 1 \mathrm{H}, J=7.58$ $\mathrm{Hz}, \mathrm{Ar}-\mathrm{H}), 7.10$ (d, 1H, J=7.58 Hz, Ar-H), $7.50(\mathrm{~s}, 1 \mathrm{H},=\mathrm{C}-\mathrm{H}), 2.18\left(\mathrm{~s}, 3 \mathrm{H}, \mathrm{CH}_{3}\right), 2.10$ $\left(\mathrm{s}, 3 \mathrm{H}, \mathrm{CH}_{3}\right)$

7.88 (d, 2H, J=8.60 Hz, Ar-H), 6.85 (d, $2 \mathrm{H}, J=8.60 \mathrm{~Hz}, \mathrm{Ar}-\mathrm{H}), 7.20(\mathrm{~d}, 1 \mathrm{H}, J=7.52$ $\mathrm{Hz}, \mathrm{Ar}-\mathrm{H}), 7.10$ (d, 1H, J=7.52 Hz, Ar-H), $7.30(\mathrm{~s}, 1 \mathrm{H},=\mathrm{C}-\mathrm{H}), 3.40\left(\mathrm{~s}, 3 \mathrm{H}, \mathrm{OCH}_{3}\right)$, $2.10\left(\mathrm{~s}, 3 \mathrm{H}, \mathrm{CH}_{3}\right), 2.02\left(\mathrm{~s}, 3 \mathrm{H}, \mathrm{CH}_{3}\right)$
Mass (m/z)

$192\left(\mathrm{M}^{+} ; 88\right), 175$ (94), 160

(100),147 (42), 132 (32), 120 (56), 105 (37), 91(38),77 (58)

$175\left(\mathrm{M}^{+} ; 96\right), 147$ (46), 119 (100), 118(58), 104 (34), 91 (24), 77 (12), 74 (8), 65 (16)

$248\left(\mathrm{M}^{+}: 12\right), 264$ (46), 220 (38), 161 (72), 133 (42), 118 (58), 91 (100), 77(80), 65(56), 29 (64)

$332\left(\mathrm{M}^{+} ; 22\right), 318$ (10), 304 (18), 290 (30), 276 (72), 248 (100), 234 (42), 205 (50), 191 (48), 177 (80), 163 (56), 130 (62), 103 (42), 91 (64)

$230\left(\mathrm{M}^{+} ; 60\right), 201(48), 186$ (100), 173(22), 155 (62), 130 (58), 91 (60), 77 (80)

$256\left(\mathrm{M}^{+} ; 10\right), 242(56), 210$ (100), 197(46), 171 (52), 156 (58), 154 (60), 140 (72), 130 (18), $102(42)$

$347\left(\mathrm{M}^{+} 81 / 79 ; 4\right), 319$ (20), 242 (12), 147 (46), 133(100), 105 (68), 91 (80), 77 (58), 65 (40)

$378\left(\mathrm{M}^{+} ; 10\right), 350$ (12), 338 (48), $326(30), 295$ (22), 219 (68), 214 (86), 188 (60), 160 (42),140 (100), 109 (58), 91 (40)

$329\left(\mathrm{M}^{+} 81 / 79 ; 2\right), 297$ (10), 271 (12), 220 (78), 211 (42), 192 (80), 185 (38), 134(100), 120 (10), 91 (28)

$360\left(\mathrm{M}^{+} ; 60\right), 328$ (30), 297 (40), 242 (38), 221 (28), 212 (74), 193 (100), 186 (60), 135 (62) 
Table II

Physical constants and \%yield of synthesized compounds (2-8)

\begin{tabular}{|c|c|c|c|}
\hline Compound No. & Melting temperature $\left({ }^{\circ} \mathrm{C}\right)$ & Color & \%Yield \\
\hline 2 & $210-211$ & Red crystalline solid & 75 \\
\hline 3 & $217-218$ & Yellow crystalline solid & 77 \\
\hline 4 & $163-164$ & Pale yellow solid & 86 \\
\hline 5 & $275-276$ & Orange red crystalline solid & 69 \\
\hline 6 & $256-257$ & Orange red crystalline solid & 62 \\
\hline 7 & $274-275$ & Yellowish crystalline solid & 77 \\
\hline $7^{\prime}$ & $255-256$ & Paleyellow crystalline solid & 77 \\
\hline 8 & $261-262$ & Dark brown crystalline solid & 85 \\
\hline $8^{\prime}$ & $276-278$ & Dark brown crystalline solid & 87 \\
\hline
\end{tabular}

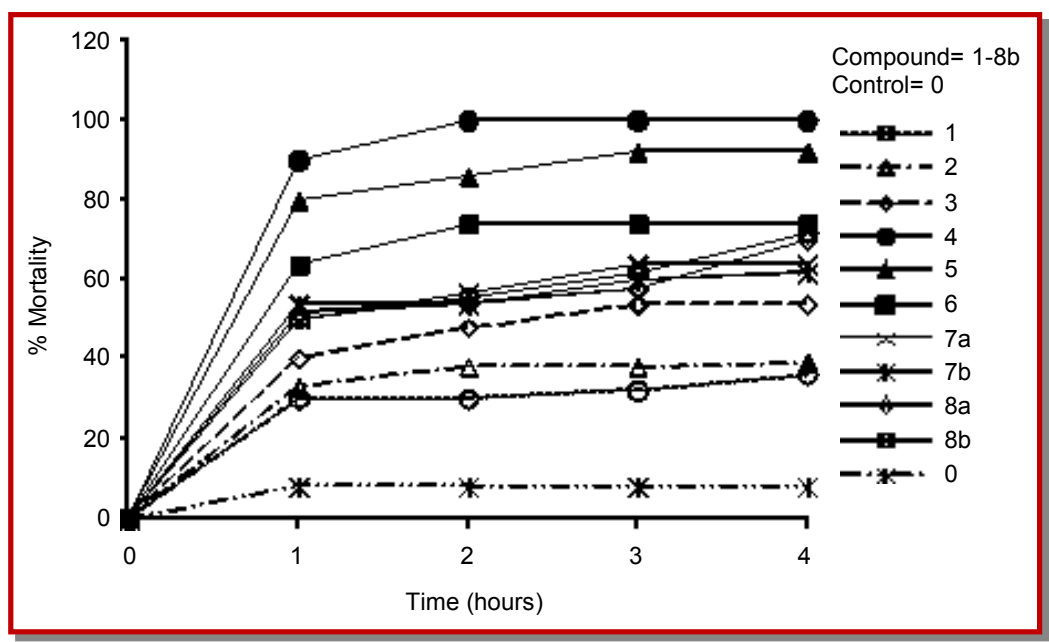

Figure 1: Brine shrimp lethality of different compounds in different incubation time

\begin{tabular}{|c|c|c|c|c|c|}
\hline \multicolumn{6}{|c|}{ Table III } \\
\hline \multicolumn{6}{|c|}{ Cytotoxicity of the synthesized compounds } \\
\hline Compound No. & $\begin{array}{l}\text { LD }_{50} \text { value } \\
\text { (ppm) }\end{array}$ & Remarks & Compound no. & $\begin{array}{l}\text { LD }_{50} \text { value } \\
\text { (ppm) }\end{array}$ & Remarks \\
\hline 1 & 99 & Less active & 6 & 22 & Highly active \\
\hline 2 & 96 & Less active & 7 & 36 & Moderately active \\
\hline 3 & 52 & Weakly active & $7^{\prime}$ & 31 & Moderately active \\
\hline 4 & 24 & Highly active & 8 & 29 & Moderately active \\
\hline 5 & 24 & Highly active & $8^{\prime}$ & 24 & Highly active \\
\hline
\end{tabular}

\section{Results}

The structures of the synthesized compounds were proved by analyzing the spectroscopic data. The results of the IR, ${ }^{1} \mathrm{H}-\mathrm{NMR}$ and MS spectra are given in the Table I. From the cytotoxicity study it was seen that compounds 4, 5, 6 and $8^{\prime}$ were highly active whereas compounds 7 , $7^{\prime}$ and 8 were moderately active and the compound 3 showed poor activity against brine shrimp. The rest of the compounds $\mathbf{1}$ and $\mathbf{2}$ were comparatively inactive.

\section{Discussion}

The structure activity relationship study of the synthesized compounds showed that dimetyl isatin along with its precursor was almost inactive. But when it was converted to Schiff base of thiosemicarbizide, it showed moderate activity. Thus sulfur atom played an important role for bioactivity.

Further cyclization of compound 3 showed greater activity. Besides this, a number of compounds containing 
several types of ring systems were synthesized from dimethyl isatin for studying the effect of extended cycle on bioactivity. An important finding of the present study showed that the greater the number of cycles adjacent to the isatin ring, the higher was the cytotoxicity (compound 4, 3 rings, $\mathrm{LD}_{50} 24$ ppm; compound 5, 3 rings, LD 5024 ppm; compound 6, 4 rings, LD 5022 ppm). But compounds containing rings far apart from the isatin ring system showed lower activity (compound 7 and $\left.7^{\prime}\right)$.

Selvam et al., 2006 showed that brominated isatin derivatives had higher activity but this study showed that it was the methoxy group which showed greater activity (compound 7, LD 5036 ppm; compound 7', LD 5031 ppm and compound 8, LD 50 ppm; compound 8', LD 2924 ppm).

\section{Acknowledgement}

We express our gratitude and thanks to Prof. Helmut Duddeck, Institute of Organic Chemistry, Hannover University, Germany for kindly supplying the MS and NMR spectra of the synthesized compounds.

\section{References}

Friebolin H. Basic one and two-dimensional NMR spectroscopy. $3^{\text {rd }}$ ed, Wiley-VCH, Wineheim, Germany, 1998, p 47.

Islam MR, Abedin J, Khayer K. Synthesis of some 5-spiro-(5/methylisatin)-4-N-acetyl-2-acetylamino- $\Delta^{2}-1,3,4$-thiadiazolines and 5-spiro-(5/-methylisatin)-4-N-acetyl-2-(5-methylisatin-3-hydrazino)- $\Delta^{2}-1,3,4$-thiadiazolines. Indian $\mathrm{J}$ Chem. 2001; 40B: 240.

Islam MR, Abedin MJ, Hossain MM, Duddeck H. Synthesis of 1-methyl bis-dioxopyrolino $[2,3: 2,3: 2,3: 6,5]$ benzene and its heterocycles via thiocarbohydrazone, thiosemicarbazone. J Bangladesh Chem Soc. 1998; 11: 71-78.

Islam MR, Khan MSA, Debnath D, Khan MMR. Synthesis of some aldehyde- $\Delta^{2}-1,3,4$-thiadiazolines heterocycles. J
Bangladesh Chem Soc. 2003; 16: 124-32.

Islam MR, Khayer K, Mahmud MI, Lingkon MH, Islam R, Duddeck $\mathrm{H}$. Cyclization of some isatin-3-thiosemicarbazones leading to $\Delta^{2}-1,3,4$-thiadiazolines and their selective deacetylation with hydrazine hydrate. J Bangladesh Chem Soc. 2000; 13: 149 .

Islam MR, Muhsin M. Synthesis of 5-chloroisatin-6,7-dimethylisatin and their carbohydrazone $\Delta^{2}-1,3,4$-oxadiazoline and their cytotoxicity studies. Bangladesh J Pharmacol. 2007; 2; 7 12.

Kondrashova NN, Shvekhgeimer MGA. A new general method for the synthesis of 2,6'-diquinoline derivatives, Doklady Chem. 2002; 2: 275.

Pal R, Jain K, Gupta GD, Handa RN, Puzari HK. Synthetic methods using isatin and derivatives. Indian J Chem. 1991; 30B: 1098.

Raj AA, Ragunathan R, Sridevikumari MR, Raman R, Synthesis, antimicrobial and antifungal activity of a new class of spiro pyrolidines. Bioorg Med Chem. 2003; 407: 11.

Ram VJ, Raj AA, Ragunathan R, Sridevikumari MR. Studies on condensed triazines as chemotherapeutic agents, II. Synthesis of 1,2,4-triazino[5,6-b]indoles and related compounds. Arch Pharmacol. 1980; 108: 313.

Selvam P, Chandramohan M, De Clereq E, Pannecouque C, Witrouw M. Synthesis and anti-HIV activity of $4-[(1,2-$ dihydro-2-oxo-3H-indol-3-ylidene)amino]-N-(4,6-dimethyl-2 -pyrimidinyl)-benzene sulphonamide and its derivatives. Eur J Pharma Sci. 2001; 14, 313-16.

Selvam P, Murugesh N, Chandramohan M, De Clereq E. Pharmacological evaluation of some novel isatin derivatives. Indian J Pharma Sci. 2004; 66: 465-68.

Selvam P, Murugesh N, Chandramohan M, Sidwell RW, Wandersee MK, Smee DF. Anti-influenza virus activity of 4[(1,2-dihydro-2-oxo-3H-indol-3-ylidene)amio]-N-(4,6-dimethyl-2-pyrimidin-2-yl)benzenesulphonamide and its derivatives. Antiviral Chem Chemotherapy. 2006 (in press).

Solis PN, Wright CW, Anderson MM, Gupta MP, Phillipson JD. A microwell cytotoxicity assays using Artima Salina (brine shrimp). Planta Med. 1993; 59: 250-52. 